\title{
Growth of vertically aligned carbon nanotubes over self-ordered nano-porous alumina films and their surface properties
}

\author{
Kuldeep Rana ${ }^{a}$, Gokce Kucukayan-Dogu ${ }^{b}$, Erman Bengu ${ }^{\text {a,* }}$ \\ a Department of Chemistry, Bilkent University, 06800 Ankara, Turkey \\ ${ }^{\mathrm{b}}$ Institute of Engineering and Science, Materials Science and Nanotechnology Graduate Program, Bilkent University, 06800 Ankara, Turkey
}

\section{A R T I C L E I N F O}

\section{Article history:}

Received 27 January 2012

Received in revised form 31 March 2012

Accepted 2 April 2012

Available online 6 April 2012

\section{Keywords:}

Anodization

Porous alumina film

Chemical vapor deposition

Carbon nanotube

Contact angle

\begin{abstract}
A B S T R A C T
Nanoporous anodic aluminum oxide (AAO) with self-organized arrays of uniform nanopores have been used for various applications in the fields of sensing, storage, separation and template-based fabrication of metal nanowires, carbon nanotubes, oxides and polymers. The work presented here involves the production and use of AAO templates for growth of aligned multi walled carbon nanotube arrays. AAO templates were formed by electrochemical oxidation of aluminum in different electrolyte solutions containing sulfuric, oxalic and phosphoric acid. SEM was used for the analysis of the surface morphology of the AAO films. The porous structures with pore size in the range of 25-120 nm were observed. Pore sizes were correlated with the type of acidic solutions used as the electrolyte. Finally, AAO surfaces have been used as substrates for the growth of vertically aligned carbon nanotubes through chemical vapor deposition technique, which showed super-hydrophobic behavior as confirmed by contact angle measurements.

(C) 2012 Elsevier B.V. All rights reserved.
\end{abstract}

\section{Introduction}

Various nanostructure based materials are already finding applications in the industry, such as electronic devices [1], biosensors [2], photonics [3], materials for energy storage [4] and as separation membranes for bio-materials [5]. Anodized aluminum oxide (AAO) template based synthesis has been employed [6] as one of the well-known production techniques enabling control of morphology, patterning and size of nanomaterials. Through its pore size, density and distribution, AAO templates have significant influence on the final properties of the nanostructured materials and thus the various structural properties of nanomaterials can be engineered by these "knobs" [4].

Discovery of self-ordered AAO membranes was first reported by Masuda and Fukuda [7-9], which, have grown by electrochemical oxidation of aluminum $(\mathrm{Al})$ and used in numerous applications in the following years [8]. The structure of porous alumina can be described as a close-packed array of columnar cells, each containing a central pore of which the size and interval can be controlled by changing the synthesis conditions [10]. AAO with a hexagonal arrangement stable at high temperature has become a popular template system for the synthesis of various functional nanostructures [5,11,12]. Furthermore, AAO films find potential applications in diverse fields as high density magnetic storage [13],

\footnotetext{
* Corresponding author. Tel.: +90 3122902153; fax: +90 3122664068.

E-mail address: bengu@fen.bilkent.edu.tr (E. Bengu).
}

DNA translocation [14] and in tribology as coatings with controlled lubricant release reservoirs [15]. Recently, anodization of Al films deposited on metal or semiconductor substrates are also studied to fabricate nanostructures on substrates using the porous AAO film as a mask [16].

The synthesis of self-organized ordered structures by anodizing of Al has been widely reported from oxalic [7,10,17], phosphoric $[5,18]$ and sulfuric acid solutions $[19,20]$. A thick and porous layer of aluminum oxide (up to $200 \mu \mathrm{m}$ ) can be formed by anodization in a dilute acidic solution. By the application of a potential difference between the electrodes, hydrogen ions are reduced to produce hydrogen gas at cathode surface and $\mathrm{Al}$ is oxidized into $\mathrm{Al}^{3+}$. A portion of the cations is dissolved in the electrolyte and the rest forms an oxide layer on the metal surface. The pore structure of AAO (size, density and distribution) can be controlled by changing the voltage, current density and acid concentration in the bath during process [21].

Some of the studies on AAO films produced by the method outlined above are targeting applications related to the usage of AAO ordering as template for the growth of carbon nanotube (CNT) arrays [22], nanowires and patterned structures [23]. Besides, inducing a pattern, AAO templates with CNTs might be used also as hybrid structures. Similar AAO/CNT hybrid structures have been showed to have potential in the following applications such as membrane [24], catalyst support [25], drug delivery [26-28] and field emitters [29].

The main aim of the present work is to control of pore size and distribution over AAO layers by changing the electrolyte bath 
type and voltage. The wetting behavior properties of these films which are a function of pore size distribution have been studied by analyzing the contact angle of de-ionized water on the AAO films. Furthermore, a thermally deposited Al film of $400 \mathrm{~nm}$ thickness over Si substrate was anodized to demonstrate the versatility of the technique used. AAO films over $\operatorname{Si}(100)$ substrate were also used for the growth of aligned CNTs by a chemical vapor deposition (CVD) technique. Similar structures have been used for building hybrid electronic devices such as field emission organic light emitting diodes [30]. In this work, we strive to demonstrate reasonable pore size control of AAO films which could enable their use as suitable templates for composite AAO/CNT structures.

\section{Experimental procedure}

Al foils of $300 \mu \mathrm{m}$ thicknesses (99.9\%, Merck) were cut in rectangles $(70 \mathrm{~mm} \times 15 \mathrm{~mm})$ and then annealed under inert atmosphere at $450^{\circ} \mathrm{C}$ for $5 \mathrm{~h}$. The annealed $\mathrm{Al}$ foils were washed with acetone and double distilled water in order to remove dirt and then dried using dry air blower. The back surface and the edges of the samples were covered by the insulating tape. The anodization of these samples were carried out by a two-step anodization procedure under constant cell voltages of $25 \mathrm{~V}, 40 \mathrm{~V}$ and $100 \mathrm{~V}$ for three different electrolyte solution baths (sulfuric, oxalic and phosphoric acid, respectively) at the temperature of $10^{\circ} \mathrm{C}$. The electrolyte baths were kept over magnetic stirrer for continuous stirring of solution to maintain the uniform concentration in the bath during the anodization step. After $60 \mathrm{~min}$ of first anodization, initial aluminum oxide formed on $\mathrm{Al}$ film was removed by chemical etching in a mixture of phosphoric ( $6 \mathrm{wt} \%$ ) and chromic acid (1.8 wt\%) at $70^{\circ} \mathrm{C}$. Immediately following the oxide removal step, Al foil was re-anodized for $2 \mathrm{~h}$ under identical conditions used for the first anodization step. Anodization of Al deposited over the Si( 100$)$ substrate was also carried out in an identical manner as used for pure Al foils.

Co-Al catalyst solution ( $5 \mathrm{mmol} / \mathrm{L}$ ) was prepared by dissolving $\mathrm{Al}\left(\mathrm{NO}_{3}\right)_{3} \cdot 9 \mathrm{H}_{2} \mathrm{O}$ and $\mathrm{Co}\left(\mathrm{NO}_{3}\right)_{2} \cdot 6 \mathrm{H}_{2} \mathrm{O}$ powders in ethanol $(\mathrm{Co}-\mathrm{Al}$ at $1: 1$ ) for CNT growth. The catalyst layer was applied on AAO surfaces either via drop-wise method or via dipping method using the prepared catalyst solution. In the drop-wise method, $20 \mu \mathrm{L} / \mathrm{cm}^{2}$ of Co-Al based catalyst solution has been dropped over the AAO surfaces and the surfaces left for air drying. In other catalyst application method, AAO surfaces were dipped into the catalyst solution at $50^{\circ} \mathrm{C}$ for $15 \mathrm{~min}$ and left for air drying. The AAO surfaces with $\mathrm{Co}-\mathrm{Al}$ catalyst loaded to the CVD chamber for CNT growth process. The reduction step proceeded under $\mathrm{H}_{2}$ and $\mathrm{Ar}$ atmospheres (flow rates $20 \mathrm{sccm}$ and $150 \mathrm{sccm}$, respectively) at $600^{\circ} \mathrm{C}$ for $15 \mathrm{~min}$. Following this step, the CNT growth was performed at the same temperature and gas mixtures with pure ethanol as a carbon source for $30 \mathrm{~min}$. The aligned CNTs have been grown over the AAO surfaces by CVD at $600{ }^{\circ} \mathrm{C}$ similar to our previous technique [32].

The structural characterization of AAO nanostructures was performed by using a Carl-Zeiss EVO 40 scanning electron microscope (SEM). The contact angle measurements have been carried out on these surfaces (Dataphysics OCA 15 plus). The synthesized carbonaceous material over AAO substrate was characterized by using different characterization techniques such as SEM, contact angle measurement and Raman spectroscopy (Horiba Jobin-Jvon-532 nm wavelength).

\section{Results and discussion}

The oxidation of Al during anodization process has been monitored by the measured oxidation current versus time as shown in Fig. 1. The measured current between electrodes is higher at
Table 1

The pore diameter obtained by using different electrolyte bath.

\begin{tabular}{lcl}
\hline Electrolyte & Voltage $(\mathrm{V})$ & $\begin{array}{l}\text { Average pore } \\
\text { diameter }(\mathrm{nm})\end{array}$ \\
\hline $\mathrm{H}_{3} \mathrm{PO}_{4}$ & 120 & 100 \\
$\mathrm{H}_{2} \mathrm{C}_{2} \mathrm{O}_{4}$ & 40 & 40 \\
$\mathrm{H}_{2} \mathrm{SO}_{4}$ & 20 & 20 \\
\hline
\end{tabular}

the beginning of the reaction which is due to higher conductivity of pure Al metal. This current decreases sharply from point A to point $B$, which is attributed to the formation of aluminum oxide barrier layer on the surface which also indicates the formation of nanopores. There is a slight increase in the current from point $B$ to point $C$ due to the decreasing thickness of the barrier layer which is caused by the increase in pore depth as shown in Fig. 1. Beyond point $C$, equilibrium is established between the competing processes of oxide formation and dissolution (pore growth). Realtime oxidation current vs. time data can be used to control/tune the oxidation period and consequently to engineer pore geometry, e.g. pore depth. Main characteristics of this behavior do not vary with the type of electrolyte bath used.

Fig. 2 shows the SEM image of top portion of the anodized Al strips prepared with three different electrolytes used (sulfuric, oxalic and phosphoric acid). Fig. 2a shows SEM image of AAO synthesized using phosphoric acid as the electrolyte which shows a uniform pore distribution all over the surface. The images from SEM show the pore distribution and their Fast Fourier Transforms (FFT) as the insets are shown. The average pore size measurements have been carried out on multiple SEM images from the AAO surfaces by employing the "particle-size analysis" option on the Image J software [31]. For Fig. 2a, the average pore diameter is found to be around $100 \pm 25 \mathrm{~nm}$ (mean \pm standard deviation) see Table 1 . The large variation for pore diameters can be attributed to the presence of high surface energy regions, such as micro-scratches on the surface of as received samples, where anodization process is accelerated resulting in larger pore diameters. Fig. $2 b$ shows the SEM micrograph of the top surface of AAO template prepared in oxalic acid electrolyte bath. The top view of pore structure after anodization shows well-ordered pore structure and the average pore diameter is found to be around $40 \pm 10 \mathrm{~nm}$. The average pore diameter obtained due to anodization in oxalic acid electrolyte bath

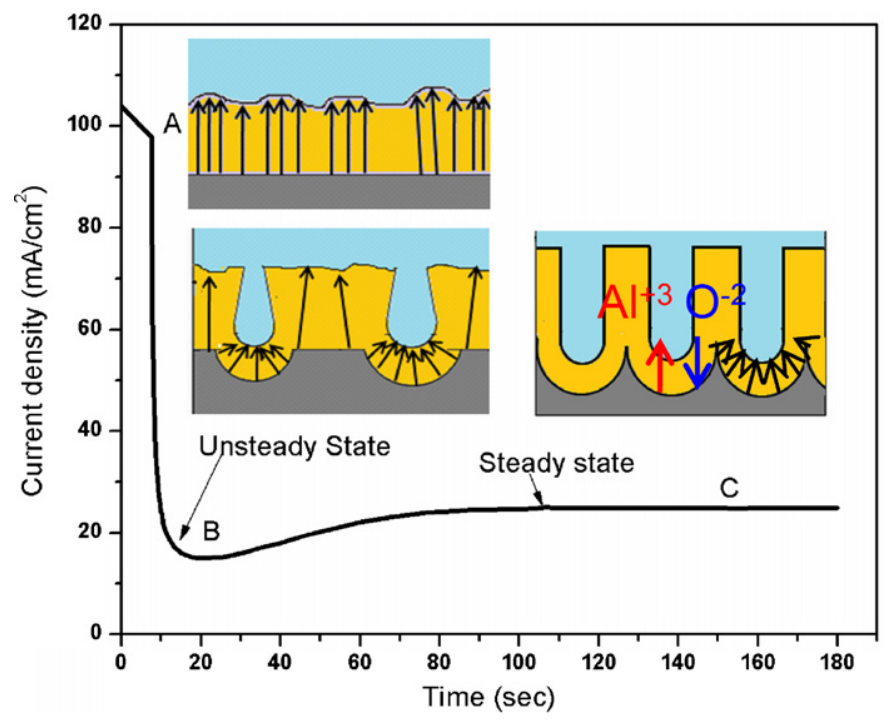

Fig. 1. Typical current density-time curve for anodization process under constantvoltage mode at a voltage of $40 \mathrm{~V}$ and in a $3 \mathrm{wt} \%$ oxalic acid bath, maintained at $15^{\circ} \mathrm{C}$. 



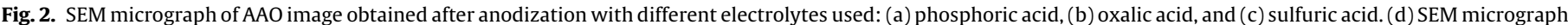
of AAO over $\mathrm{Si}$ anodized in phosphoric acid solution.

is smaller compared to that obtained using phosphoric acid bath requiring a higher voltage for pore formation. Fig. $2 c$ shows the SEM micrograph of the surface after anodization using sulfuric electrolyte; anodization has been carried out at $20 \mathrm{~V}$ for this sample. The pores are distributed in a regular hexagonal manner with an average pore diameter $20 \pm 9 \mathrm{~nm}$ as calculated. The average pore diameter obtained with sulfuric acid is smallest among all three acids used for the electrolyte bath in this study.

Further to ensure a constant pore depth across our sample, a $400 \mathrm{~nm}$ thick Al film has been deposited over Si substrate via thermal deposition method. This film was then anodized in phosphoric electrolyte bath. Fig. 2d shows the SEM image of AAO structure over Si substrate, which shows that AAO film was developed successfully and maximum pore depth can be controlled by controlling the thickness of deposited Al layer. Such films with tuned pore depths can be used for electronic application.

The pore formation occurs due to electric-field assisted oxide dissolution. At this stage the current begins to increase, due to decrease in resistance as oxide layer thickness reduces in front of the initiating pores. The current stables when a dynamic equilibrium is established between the competing mechanisms of aluminum oxide growth and its partial dissolution leading to pore formation at the surface [33]. Initially an irregular array of pore structures form on the surface as the pores are created randomly on the sample. However, due to the repulsive forces between neighboring pores a self-organized pore array eventually forms. The mechanical stress associated with the expansion of the Al during oxide formation is cited as the cause of repulsive forces between neighboring pores which leads to self-organization [10].

We investigated the self-organization behavior of the pores using the FFT images provided as the insets of Fig. 2. The FFT images were calculated from the respective SEM images using Image J software [31]. Three different patterns for pores are observed through the examination of the FFT images. The FFT images of anodized samples in phosphoric and oxalic acids show six distinct spots forming a hexagon (Fig. 2a and b). This observation indicates a single domain of well-ordered, long-range periodic 2-D lattice formed by the pores [34]. The FFT image of anodized surface in sulfuric acid shows a ring shape form (Fig. 2c). This suggests the presence of imperfections in the periodicity of the surface and multiple domains of ordered 2-D lattice of pores. As shown in Fig. 2d, the FFT image indicates a diffuse ring for the case of AAO over Si sample prepared with phosphoric acid which reveals the presence of disordered domains on the surface. In the literature, Sulka et al. [34] concluded that high stresses on the surface of $\mathrm{Al}$ can destroy the long-range arrangement of pores and hence, the exact mechanism of pore ordering is still open for debate. According to this, multiple domain formation on the anodized surface using phosphoric acid might be due to the presence of non-homogenous distribution of stresses in the Al film deposited over Si substrate.

As mentioned earlier, in some cases preparation of nanostructures using porous AAO as templates involves the introduction of dissolved materials into the pores of the membranes. One way to determine whether the dissolved solvents are going to fill in the pores is to investigate the wetting behavior of common solvents on the AAO surfaces. Hence, we investigated the contact angle of water on these AAO surfaces. The contact angle measurement shows that all three surfaces are hydrophilic in nature with initial contact angles are $59^{\circ}, 44^{\circ}$ and $57^{\circ}$ respectively for AAO sheets anodized in sulfuric, oxalic and phosphoric acid as shown in Fig. 3a. Two different value of contact angles have been observed at two ends of droplet, which shows that surface energy is varying region to region of AAO surface. Contact angle value decreases with time and after 5 min we measured $22^{\circ}, 22.7^{\circ}$ and $40^{\circ}$ respectively for sulfuric, oxalic and phosphoric acids as shown in Fig. 3b. The decrease in contact angles in all three cases after a few minutes is due to the seeping of the water drop into pores of AAO. Wetting of AAOs has been explained as follow; if a liquid is allowed to spread on the pore walls of AAOs, first the liquid is brought into contact with the AAO surfaces low-energy liquids spread rapidly on high-energy surfaces and the driving forces involved in this process are due to shortrange as well as long-range polar interactions between the wetting liquid and the pore walls [35]. After wetting the walls of nanoholes, the water dispersed rapidly into the hole as contact angle decreases very fast as shown in Fig. $3 \mathrm{~b}$. 
(a)



(b)

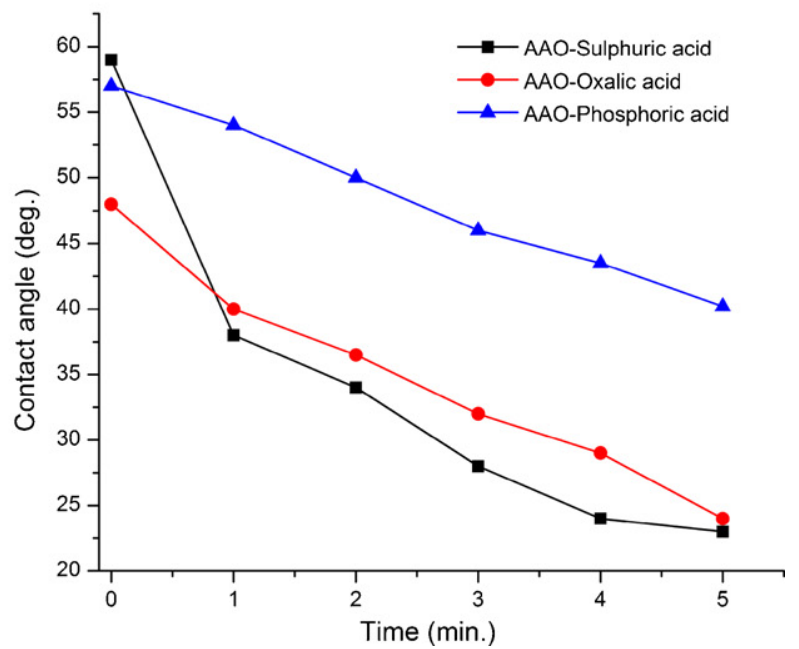

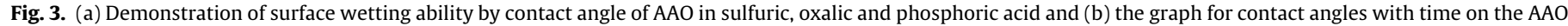
surfaces are shown.

The final section of the study involves the application of AAOs as a template for the vertically aligned CNT growth. The vertically aligned CNTs have been grown over two different templates: AAO and $\mathrm{AAO} / \mathrm{Si}$ substrates anodized in phosphoric acid. Fig. 4 shows the SEM images of CNTs grown over AAO substrate, in one case the catalyst layer was applied via drop-wise application over the AAO surface (Fig. 4a) while in other case the AAO substrate dipped into the catalyst solution for $15 \mathrm{~min}$. (Fig. 4b). SEM image of CNTs in Fig. 4a shows that CNTs are not aligned; instead they are tangled with each other as shown in Fig. 4b. Most of the CNTs are appeared on the surface and very few are coming from the pores of AAO in the drop-wise case. This shows that most of the catalysts particles stayed on the top surface of the AAO and very few penetrated through the pores. Fig. $4 \mathrm{~b}$ shows the alignment of CNTs grown on AAO substrate in which catalyst has been deposited by dipping substrate into the vial containing the catalyst solution at $50^{\circ} \mathrm{C}$ for $15 \mathrm{~min}$. The as-grown CNTs are apparently straight and parallel to each other forming a dense surface with tube height about $3 \mu \mathrm{m}$ measured from Fig. 4b. The growth of dense CNTs (Fig. 4b) indicates that the catalyst stays on the surface as well as it penetrates into the pore channels for this case, which makes it clear that the final CNT alignment is affected by the method of catalyst deposition.

CNTs were also grown on the well-ordered AAO template formed on Si substrate and SEM images are shown in Fig. 5 at two different magnifications. CNTs are very dense, vertically aligned and distributed uniformly all over the AAO surface (Fig. 5a). To demonstrate a better view for the alignment of CNTs inside the template, a cross-sectional SEM image is shown in Fig. 5b. CNTs are indeed well aligned and lengths are very close to each other about $3 \mu \mathrm{m}$ long. The roots of the CNTs are located at the bottom of the pores as shown in Fig. 5b. These results confirm that by changing the pore depth (through controlling the thickness of Al layer over $\mathrm{Si}$ ), the catalyst solution can penetrate easily inside the pore and hence the growth of aligned and dense CNTs can be achieved.

First and second order Raman spectra of CNTs grown over AAO and $\mathrm{AAO} / \mathrm{Si}$ substrate are shown in Fig. 6. The first order Raman spectra shows two intense peaks which are well known as $G$ and $\mathrm{D}$ peak (Fig. 6a). The $\mathrm{G}$ mode (TM - tangential mode) corresponds to the in-plane vibration of two atoms in a hexagonal lattice. In our study, this mode is located around $1578 \mathrm{~cm}^{-1}$ and $1584 \mathrm{~cm}^{-1}$ respectively for CNTs grown over $\mathrm{AAO} / \mathrm{Si}$ and $\mathrm{AAO}$ substrate. The D-band (disorder band is located between 1330 and $1360 \mathrm{~cm}^{-1}$ ) is expected to be observed in multi walled carbon nanotubes (MWCNTs), which is $1338 \mathrm{~cm}^{-1}$ and $1348 \mathrm{~cm}^{-1}$ respectively for CNTs grown over $\mathrm{AAO} / \mathrm{Si}$ and $\mathrm{AAO}$ substrates. First order Raman spectra of CNTs are similar for both surfaces.

Raman features are analyzed by using a Lorentzian fit to the $D$ and $G$ peaks. The ratio of integrated intensity of $G$ to D-band $\left(I_{\mathrm{G}} / I_{\mathrm{D}}\right)$ gives the degree of order in the carbonaceous material. $I_{\mathrm{G}} / I_{\mathrm{D}}$ ratio for the CNTs grown over $\mathrm{AAO} / \mathrm{Si}$ and $\mathrm{AAO}$ substrates are 0.85
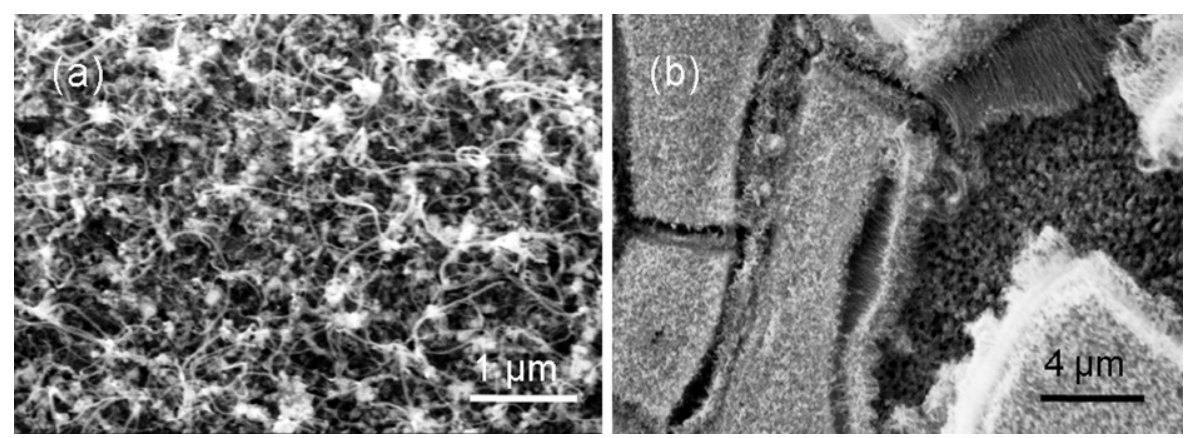

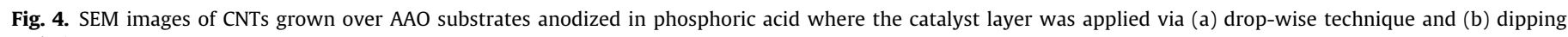
technique. 

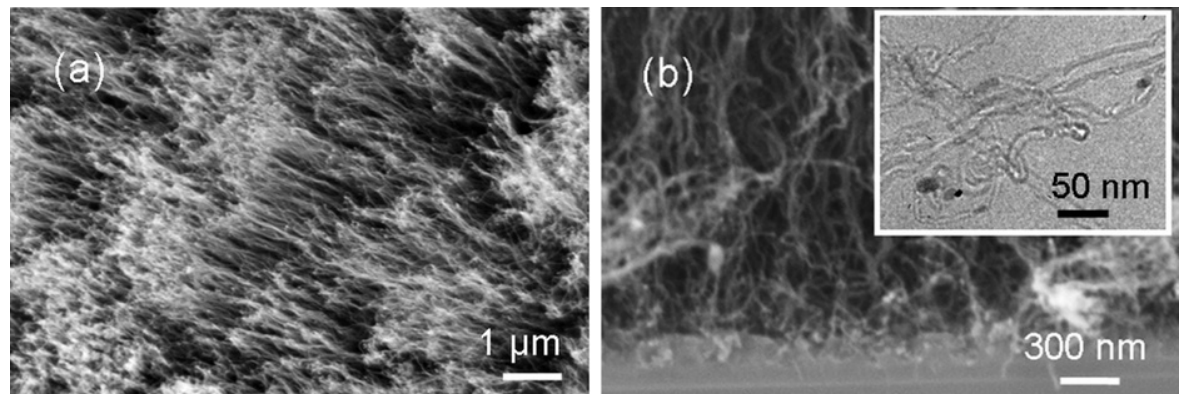

Fig. 5. (a) Top and (b) cross sectional SEM images of CNTs grown over AAO/Si substrates anodized in phosphoric acid. Inset of (b) shows the TEM image of CNTs.
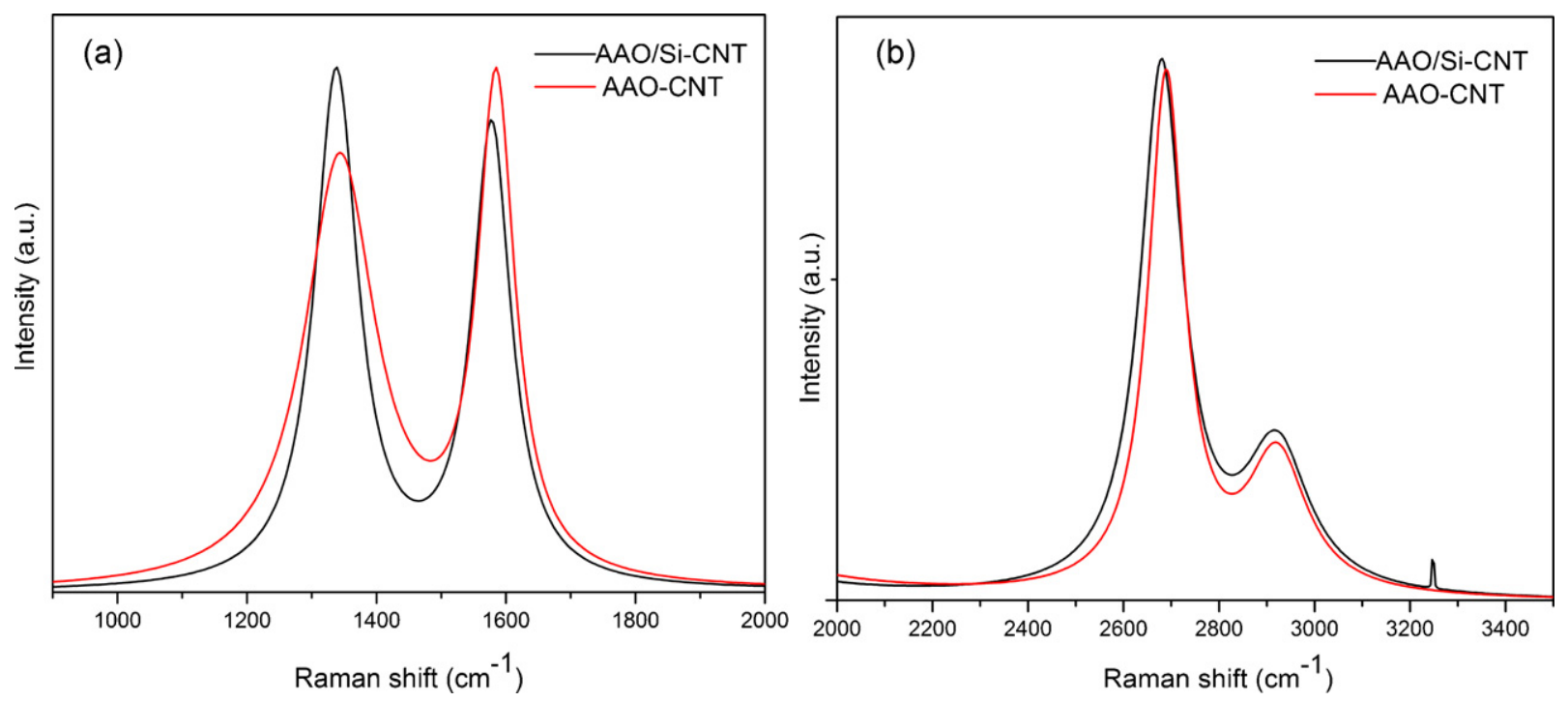

Fig. 6. (a) First order and (b) second order Raman spectra of CNTs grown over two different substrates: AAO and AAO/Si.

and 0.60 , respectively. This shows that CNTs grown over AAO/Si substrate are more ordered and less defective as compared to the CNTs over AAO substrates. The CNTs grown over AAO substrates are entangled to each other and twisted which creates more defects on CNT surface, however in other case; CNTs are straight and parallel to each other and have higher $I_{\mathrm{G}} / I_{\mathrm{D}}$ value which means fewer defects. Second order Raman spectra in the region between $2400 \mathrm{~cm}^{-1}$ and $3400 \mathrm{~cm}^{-1}$ are shown in Fig. 6b, which shows sharp and strong peak centered at $2679 \mathrm{~cm}^{-1}$ and $2689 \mathrm{~cm}^{-1}$ respectively for CNTs grown over AAO/Si and AAO substrates. These peak are assigned as $2 \mathrm{D}$ or $\mathrm{G}^{\prime}$-band $\left(2700 \mathrm{~cm}^{-1}\right)$. This band is an intrinsic property of well-ordered $\mathrm{sp}^{2}$ carbons [36] and closely related to the band structure of graphene layers in carbon. It has been reported that 2D-band further downshifts due to disorder or defects presence in carbon lattice [36]. The other band almost at similar position around
$2922 \mathrm{~cm}^{-1}$ is related to the combination of G and D-band $(G+D)$ in carbonaceous materials [37].

The wetting ability of the CNT surfaces is an important property which is governed both by the surface chemistry and the microstructure of the surface in contact with the solvent $[38,39]$. The contact angle was measured for the two different types of CNTs; aligned forest like and entangled CNTs grown over AAO/Si and AAO substrates, respectively (Fig. 7). The vertically aligned CNTs over AAO/Si substrate clearly display a superhydrophobic behavior with a contact angle of $180^{\circ}$ (Fig. 7a and see Supplementary Video) while the entangled CNTs grown over AAO substrate have contact angle around $163^{\circ}$ (Fig. 7b). Similar behavior was also reported by Wang et al. $[40,41]$ in which the contact angle values measured against water for aligned CNTs of few micrometers long and entangled CNTs were $174^{\circ}$ and $144^{\circ}$, respectively [42]. The contact angle values
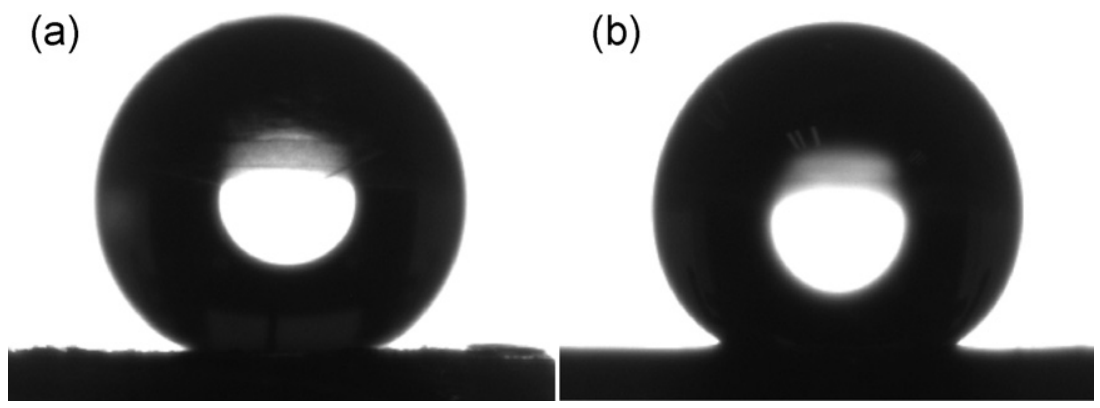

Fig. 7. Contact angle images for (a) the vertically aligned CNTs grown on AAO/Si substrate and (b) the entangled CNTs grown on AAO substrates. 
remain almost constant even after $5 \mathrm{~min}$, i.e. $178^{\circ}$ and $158^{\circ}$ respectively for the vertically aligned CNTs and the entangled CNTs. The water droplet retained its spherical shape even after $15 \mathrm{~min}$; however, elsewhere Lau et al. [41] reported after a few minutes, the water drop wetted the CNTs and seeped in the CNT forest. Thus, in our case, we did not observe any seepage of the water drop into the CNT forest which might be due to higher density of CNTs grown on the $\mathrm{AAO} / \mathrm{Si}$ surface.

The clear difference in the measured contact angles of the vertically aligned CNTs and the entangled CNTs can be explained by an assumption made by Pavese et al. [42] which states that superhydrophobic behavior depends on the actual contact surface area between the water drop and CNTs. Thus, in the case of vertically aligned CNTs, the actual contact surface area is very small limited to the tips of CNTs. In the case of the entangled CNTs, the measured value of contact angle is lower $\left(163^{\circ}\right)$ because of the disorder in the alignment of the CNTs with respect to the substrate surface and the side-walls of the misaligned CNTs increasing the total contact area.

\section{Conclusions}

In this study, we have synthesized AAO templates with different pore sizes using various electrolyte baths. To understand the wettability/pore filling behavior, contact angle measurements have been carried out which show hydrophilic natures of these surfaces. This result has a practical importance showing that water soluble materials could be easily utilized for filling the porous alumina templates. For instance, using ethanol based catalyst precursor solutions we were able to successfully grow aligned and dense CNT forests over both AAO and AAO/Si substrates. Such hybrid structures combining patterned conductor and insulator arrays can be potentially applied in the electronics industry as structures for building light emitting diodes, solar cells and super capacitors.

\section{Acknowledgements}

G. Kucukayan-Dogu thank the Scientific and Technological Research Council of Turkey (Tubitak) for financial support. This work was partially supported by Tubitak Projects 109 T026 and $107 \mathrm{~T} 892$.

\section{Appendix A. Supplementary data}

Supplementary data associated with this article can be found, in the online version, at http://dx.doi.org/10.1016/j.apsusc.2012.04.008.

\section{References}

[1] J. Goldberger, R. He, Y. Zhang, S. Lee, H. Yan, H.-J. Choi, P. Yang, Single-crystal gallium nitride nanotubes, Nature 422 (2003) 599-602.

[2] P. Kohli, M. Wirtz, C.R. Martin, Nanotube membrane based biosensors, Electroanalysis 16 (2004) 9-18.

[3] J.S. King, D. Heineman, E. Graugnard, C.J. Summers, Atomic layer deposition in porous structures: 3D photonic crystals, Appl. Surf. Sci. 244 (2005) 511-516.

[4] P. Banerjee, I. Perez, L. Henn-Lecordier, S.B. Lee, G.W. Rubloff, Nanotubular metal-insulator-metal capacitor arrays for energy storage, Nat. Nanotechnol. 4 (2009) 292-296.

[5] S.B. Lee, D.T. Mitchell, L. Trofin, T.K. Nevanen, H. Söderlund, C.R. Martin, Antibody-based bio-nanotube membranes for enantiomeric drug separations, Science 296 (2002) 2198-2200.

[6] B. Nandan, B.K. Kuila, M. Stamm, Supramolecular assemblies of block copolymers as templates for fabrication of nanomaterials, Eur. Polym. J. 47 (2011) 584-599.

[7] H. Masuda, K. Fukuda, Ordered metal nanohole arrays made by a two-step replication of honeycomb structures of anodic alumina, Science 268 (1995) 1466-1468.

[8] G.E. Thompson, G.C. Wood, Porous anodic film formation on aluminium, Nature 290 (1981) 230-232.

[9] R.C. Furneaux, W.R. Rigby, A.P. Davidson, The formation of controlled-porosity membranes from anodically oxidized aluminium, Nature 337 (1989) 147-149.
[10] O. Jessensky, F. Muller, U. Gosele, Self-organized formation of hexagonal pore arrays in anodic alumina, Appl. Phys. Lett. 72 (1998) 1173-1175.

[11] S. Park, J.-H. Lim, S.-W. Chung, C.A. Mirkin, Self-assembly of mesoscopic metalpolymer amphiphiles, Science 303 (2004) 348-351.

[12] N.I. Kovtyukhova, T.E. Mallouk, Nanowire p-n heterojunction diodes made by templated assembly of multilayer carbon-nanotube/polymer/semiconductorparticle shells around metal nanowires, Adv. Mater. 17 (2005) 187-192.

[13] R. Karnik, R. Fan, M. Yue, D.Y. Li, P.D. Yang, A. Majumdar, Electrostattic control of ions and molecules in nanofluidic transistors, Nano Lett. 5 (2005) 943-947.

[14] A. Meller, L. Nivon, D. Branton, Voltage-driven DNA translocations through a nanopore, Phys. Rev. Lett. 86 (2001) 3435-3438.

[15] G.-S. Lee, J.H. Choi, Y.C. Choi, S.D. Bu, Y.-Z. Lee, Tribological effects of pores on an anodized Al alloy surface as lubricant reservoir, Curr. Appl. Phys. 11 (2011) S182-S186.

[16] Y. Kanamori, K. Hane, H. Sai, H. Yugami, $100 \mathrm{~nm}$ period silicon antireflection structures fabricated using a porous alumina membrane mask, Appl. Phys. Lett. 78 (2001) 142-143.

[17] L. Ba, W.S. Li, Influence of anodizing conditions on the ordered pore formation in anodic alumina, J. Phys. D 33 (2000) 2527-2531.

[18] H. Masuda, M. Ohya, K. Nishio, H. Asoh, M. Nakao, M. Nohtomi, A. Yakoo, T. Tamamura, Photonic band gap in anodic porous alumina with extremely high aspect ratio formed in phosphoric acid solution, Jpn. J. Phys. Part 239 (2000) 1039-1041.

[19] W.Y. Zhou, Y.B. Li, Z.Q. Liu, D.S. Tang, X.P. Zou, G. Wang, Self-organized formation of hexagonal nanopore arrays in anodic alumina, Chin. Phys. 10 (2001) $218-222$.

[20] G.C. Wood, J.P. O'Sullivan, The anodizing of aluminium in sulphate solutions, Electrochim. Acta 15 (1970) 1865-1876

[21] G.D. Sulka, K.G. Parkoa, Anodising potential influence on well-ordered nanostructures formed by anodisation of aluminium in sulphuric acid, Thin Solid Films 515 (2006) 338-345.

[22] J. Li, C. Papadopoulos, J.M. Xu, M. Moskovits, Highly-ordered carbon nanotube arrays for electronics applications, Appl. Phys. Lett. 75 (1999) 367-369.

[23] L. Piraux, A. Encinas, L. Vila, S. Matefi-Tempfli, M. Matefi-Tempfli, M. Darques, F. Elhoussine, S. Michotte, Magnetic and superconducting nanowires, J. Nanosci. Nanotechnol. 5 (2005) 372-389.

[24] Z. Wang, F. Hu, P.K. Shen, Carbonized porous anodic alumina as electrocatalyst support for alcohol oxidation, Electrochem. Commun. 8 (2006) 1764-1768.

[25] S. Sigurdson, V. Sundaramurthy, A.K. Dalai, J. Adjaye, Effect of anodic alumina pore diameter variation on template-initiated synthesis of carbon nanotube catalyst supports, J. Mol. Catal. A: Chem. 306 (2009) 23-32.

[26] H. Hillebrenner, F. Buyukserin, J.D. Stewart, C.R. Martin, Template synthesized nanotubes for biomedical delivery applications, Nanomedicine (Lond.) 1 (2006) 39-50.

[27] J.L. Perry, R. Martin, J.D. Stewart, Drug-delivery strategies by using templatesynthesized nanotubes, Chem.-Eur. J. 17 (2011) 6296-6302.

[28] S. Kataoka, T. Yamamoto, Y. Inagi, A. Endo, M. Nakaiwa, T. Ohmori, Synthesis of ordered mesoporous carbon thin films at various temperatures in vapor infiltration method, Carbon 46 (2008) 1358-1360.

[29] G. Che, B.B. Lakshmi, E.R. Fisher, C.R. Martin, Carbon nanotube membranes for electrochemical energy storage and production, Nature 393 (1998) 346-349.

[30] C.-S. Li, S.-H. Su, H.-Y. Chi, M. Yokoyama, Application of highly ordered carbon nanotubes templates to field-emission organic light-emitting diodes, J. Cryst. Growth 311 (2009) 615-618.

[31] M.D. Abramoff, P.J. Magalhaes, S.J. Ram, Image processing with ImageJ, Biophoton. Int. 11 (2004) 36-42.

[32] B. Baykal, V. Ibrahimova, G. Er, E. Bengü, D. Tuncel, Dispersion of multi-walled carbon nanotubes in an aqueous medium by water-dispersible conjugated polymer nanoparticles, Chem. Commun. 46 (2010) 6762-6764.

[33] G.E. Thompson, Porous anodic alumina: fabrication, characterization and applications, Thin Solid Films 297 (1997) 192-201.

[34] G.D. Sulka, S. Stroobants, V.V. Moshchalkov, G. Borghs, J.-P. Celis, Effect of tensile stress on growth of self-organized nanostructures on anodized aluminum, J. Electrochem. Soc. 151 (2004) B260-B264.

[35] R. Redon, A. Vazquez-Olmos, M.E. Mata-Zamora, A. Ordonez-Medrano, F. Rivera-Torres, J.M. Saniger, Contact angle studies on anodic porous alumina, J. Colloid. Interface Sci. 287 (2005) 664-670.

[36] P.C. Eklund, J.M. Holden, R.A. Jishi, Vibrational modes of carbon nanotubes: spectroscopy and theory, Carbon 33 (1995) 959-972.

[37] H. Maeta, Y. Sato, Raman spectra of neutron-irradiated pyrolytic graphite, Solid State Commun. 23 (1997) 23-25.

[38] P. Kim, C.M. Lieber, Nanotube nanotweezers, Science 286 (1999) 2148-2150.

[39] B.J. Hinds, N. Chopra, T. Rantell, R. Andrews, V. Gavalas, L.G. Bachas, Aligned multiwalled carbon nanotube membranes, Science 303 (2004) 62-65.

[40] Z. Wang, L. Ci, L. Chen, S. Nayak, P.M. Ajayan, N. Koratkar, Polarity-dependent electrochemically controlled transport of water through carbon nanotube membranes, Nano Lett. 7 (2007) 697-702.

[41] K.K.S. Lau, J. Bico, K.B.K. Teo, M. Chhowalla, G.A.J. Amaratunga, W.I. Milne, G.H. McKinley, K.K. Gleason, Superhydrophobic carbon nanotube forests, Nano Lett. 3 (2003) 1701-1705.

[42] M. Pavese, S. Musso, S. Bianco, M. Giorcelli, N. Pugno, An analysis of carbon nanotube structure wettability before and after oxidation treatment, J. Phys. Condens. Matter 20 (2008) 1-7. 